\title{
Tolerance-Maps for line-profiles constructed from Boolean intersection of T-Map primitives for arc-segments ${ }^{*}$
}

\author{
Yifei $\mathrm{HE}^{1}$, Joseph K. DAVIDSON ${ }^{\dagger 2}$, Jami J. SHAH ${ }^{\dagger 2}$ \\ ('Siemens PLM Software, Inc., 2000 Eastman Drive, Milford, Ohio 45150, USA) \\ ( ${ }^{2}$ Mechanical \& Aerospace Engineering, Arizona State University, Tempe, Arizona 85287-6106, USA) \\ †E-mail: J.Davidson@asu.edu; jami.shah@asu.edu \\ Received July 30, 2014; Revision accepted Dec. 3, 2014; Crosschecked Apr. 15, 2015
}

\begin{abstract}
For purposes of automating the assignment of tolerances during design, a math model, called the Tolerance-Map (T-Map), has been produced for most of the tolerance classes that are used by designers. Each T-Map is a hypothetical point-space that represents the geometric variations of a feature in its tolerance-zone. Of the six tolerance classes defined in the ASME/ANSI/ISO Standards, profile tolerances have received the least attention for representation in computer models. The objective of this paper is to describe a new method of construction, using computer-aided geometric design, which can produce the T-Map for any line-profile. The new method requires decomposing a profile into segments, creating a solid-model T-Map primitive for each, and then combining these by Boolean intersection to generate the T-Map for a complete line profile of any shape. To economize on length, the scope of this paper is limited to line-profiles formed from circular arc-segments. The parts containing the line-profile features are considered to be rigid.
\end{abstract}

Key words: Geometric tolerance, Line-profile, Tolerance modelling, Tolerance-zone, Boolean intersection doi:10.1631/jzus.A1400239

Document code: A

CLC number: TH161

\section{Introduction}

Geometric tolerancing is the modern method that designers use to specify allowable limits for the geometric manufacturing variations on the features of a part, variations such as size, position, form, runout, and orientation. The rules for specifying and interpreting all classes of geometric tolerances are codified in the ASME Y14.5 Standard (ASME, 2009) and the ISO standards. The ISO 1660 Standard (ISO, 1987 ) is specific to profiles. A tolerance specification defines a tolerance-zone in which the feature of interest has several degrees of freedom of

\footnotetext{
${ }^{\ddagger}$ Corresponding author

* Project supported by the National Science Foundation of the USA (No. CMMI-0969821)

(C) Zhejiang University and Springer-Verlag Berlin Heidelberg 2015
}

displacement. The location of the tolerance-zone is established with basic dimensions, and its boundaries are determined by the specified tolerance(s).

There have been several attempts to model the variations that are described in the Standards. Summaries of these, together with a comparison of the Arizona State University (ASU) Tolerance-Maps (T-Maps) model with other math models for tolerances, appear in (Pasupathy et al., 2003; Mujezinović et al., 2004; Ameta et al., 2011). The T-Maps model is one of several vector space models that map geometric manufacturing variations into a region of parametric space. Other authors have used other vector space models (Giordano et al., 1999; Roy and $\mathrm{Li}, 1999)$ to model the 3D variations of a planesegment (e.g., rectangular or circular) or the $4 \mathrm{D}$ variations of a line-segment. The conclusion of the comparison of models in (Mujezinović et al., 2004; 
Ameta et al., 2011) is that the other models contribute substantially to the science of representing geometric variations and tolerances, but that each model either is limited structurally from representing, or has not been developed to represent, one or more of the following aspects of the Standards: form tolerances, floating zones, Rule \#1 tradeoff, bonus tolerance arising from material condition, and/or datum precedence. In addition, with one exception, none of those models have ever been formulated to represent profile tolerances, either in theory or in software for computer-aided tolerancing. We know of one exception: Giordano's and Duret's use of deviation space to model a rectangular clearance space (Giordano and Duret, 1993).

Tolerances on line-profiles are used to control manufacturing variations for cross-sectional shapes of parts, even mildly twisted ones, such as those on turbine or compressor blades. Such tolerances limit geometric manufacturing variations to a 2D tolerance-zone, i.e., an area, the boundaries to which are curves parallel to the true profile. The single profile tolerance may be used to control position, orientation, and form of the profile. A profile tolerance may also be used to control local variations on a large interrupted surface (ASME, 2009). Often formed by several isolated raised bosses, such a surface commonly forms the attachment feature on many metal castings and molded plastic parts.

Our objective in this paper is to produce a math model that represents manufacturing variations of line-profiles and their limits (tolerances), yet does so in a manner consistent with their representations for other features, such as lines and planes. With such a model, tolerance chains may contain a mixture of features, even different classes of tolerances, and yet be represented in a uniform way.

As a first attempt at modeling line-profiles, T-Maps for some specific line-profile shapes were presented in (Davidson and Shah, 2012): for squares, for rectangles, and for those having an isosceles right-triangular shape. These T-Maps were simple enough that the intuitive method used there was sufficient to get an accurate result. However, since the publication of (Davidson and Shah, 2012), we have found that the boundaries of those T-Maps were doubly traced, either in large part or entirely. Minor variations to those profile shapes cause the doubly traced portions to separate into so many faces and/or curved surfaces that the intuitive method becomes burdensome.

An alternative construction method is described in (He et al., 2013). There, the authors describe how, for a scalene triangular shape, solid-model T-Map primitives, one for each line-segment, may be produced, deformed, and then combined with Boolean intersection to get the T-Map for the entire profile. The method is applicable to any polygonal shape. The purpose of this paper is to modify the method of Boolean intersection of primitive T-Maps in ( $\mathrm{He}$ et al., 2013) so that it applies to line-profiles composed only of circular arc-segments.
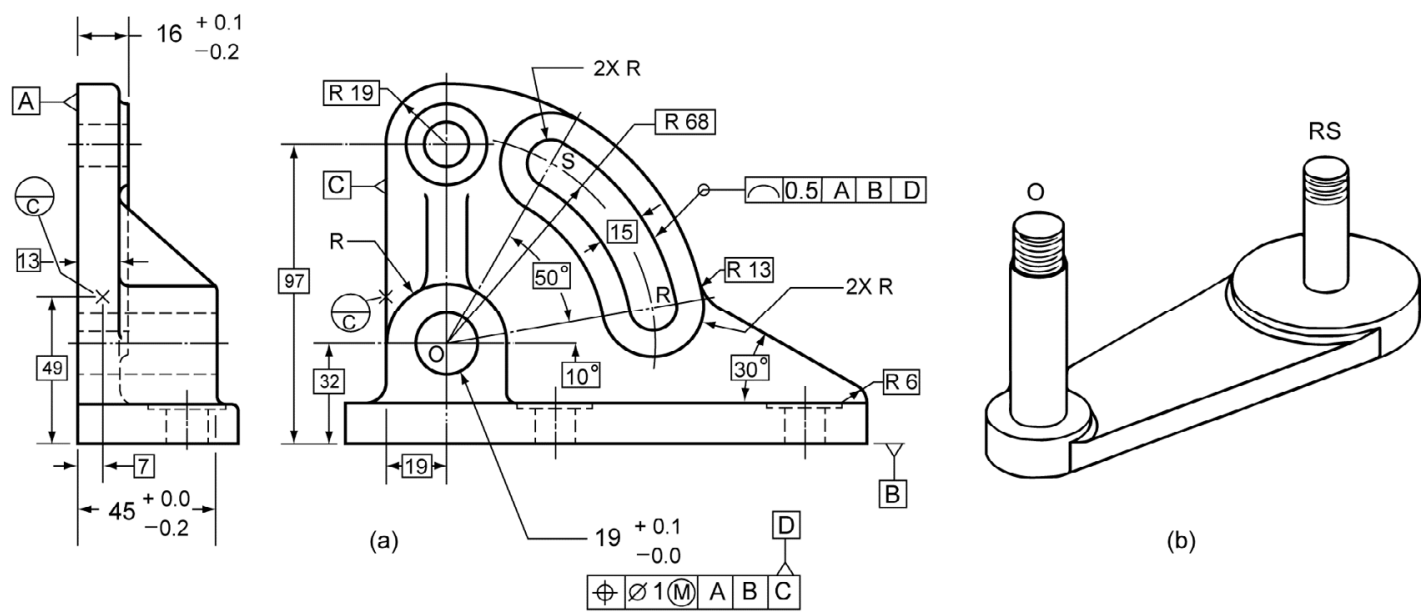

Fig. 1 (a) A partially dimensioned cast part with a hole (Datum D) and a tolerance of $t=0.5 \mathrm{~mm}$ applied to the $50^{\circ}$ adjustment arc-slot $A B$ to control its size, location, and form; (b) An adjusting-link to engage hole at $O$ and arc-slot $\overline{R S}$ 


\section{Profile specifications and tolerance-zone boundaries}

The casting in Fig. 1a contains an arc-shaped opening to permit an operator to adjust machinery by clamping a bolt at any location within the $50^{\circ}$ range shown. The opening, an arc-slot, is composed of four joined arc-segments. The theoretical finished shape for this opening is specified with the basic angle- and radius-dimensions. Geometric manufacturing variations to its machined shape are controlled by the profile tolerance $t=0.5 \mathrm{~mm}$ relative to the Datums A, B, and $\mathrm{D}$. This specification establishes two boundaries formed by parallel curves. One is $0.25 \mathrm{~mm}$ larger along every line normal to the surface, and the other is $0.25 \mathrm{~mm}$ smaller. According to the standards (ISO, 1987; ASME, 2009), measured points on the manufactured surface at every cross-section (each forming a line-profile) through the depth must lie within the outer and inner boundary lines at that cross-section.

The adjustment arc-slot in Fig. 1a is reproduced in Fig. 2 along with its tolerance-zone boundaries. The middle-sized profile (MSP) is shown with a dashed line. Note that the range of allowable angular displacement of the MSP within the tolerance-zone is determined by the ratio $t / d$, where $d$ is the longest interior linear dimension between arc-centers (length $\overline{R S}$ in Figs. 1a and 2).

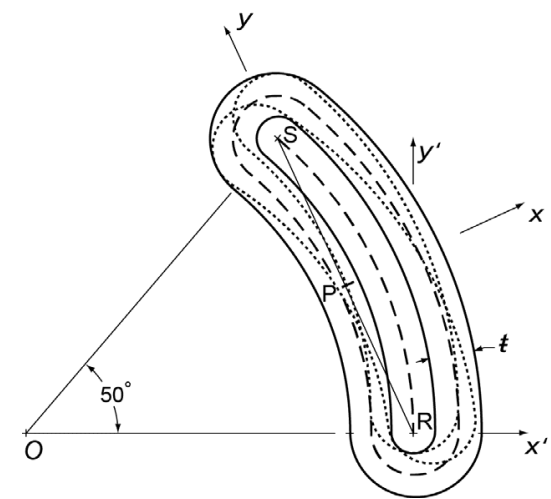

Fig. 2 The middle-sized profile (dashed curve), consisting of four joined arc-segments; the inner and outer boundaries to its tolerance-zone; its idealized profile, arc $\overline{R S}$; and two limiting displacements (dotted-lines), one upward, the other rotated counterclockwise (CCW)

\section{Tolerance-Map (T-Map) for a line-profile}

A Tolerance-Map ${ }^{\circledR}\left(\mathrm{T}-\mathrm{Map}^{\circledR}\right)$ for any feature (Davidson et al., 2005) is a hypothetical Euclidean point-space, the size and shape of which reflects all the allowable geometric variations for that feature. It is the range (co-domain) of points resulting from a one-to-one mapping from all the geometric variations from the manufacture of a feature, within its tolerance-zone, to the Euclidean point-space. These allowable manufacturing variations are displaced locations of the feature relative to its theoretical location on the part. They are limited by the boundaries to the tolerance-zone, which, for line-profiles, are dependent upon the shape of the profile, the value of tolerance(s) assigned to it, and other specifications, such as datums, in the feature control frame (Fig. 1a and ISO (1987) and ASME (2009)).

Since a T-Map represents the freedom of a feature in its tolerance-zone, the T-Map may be a bounded area (2D), volume (3D), or a hypervolume $(4 \mathrm{D}, 5 \mathrm{D}, \ldots, n \mathrm{D})$ of points. For line-profiles, the manufacturing variations are represented with the true profile, which is of perfect form, and allowable perfect-form profiles parallel to it, instead of the more familiar plane, line, or point. And, each point in the T-Map corresponds to any one of these perfect-form profiles or to any one of them that is displaced, yet remains within the tolerance-zone. Consequently, its T-Map is a 4D hypervolume, i.e., a 4D solid. The allowable manufacturing variations are: change in radius (size), two translational displacements $e_{x}$ and $e_{y}$, and one rotational displacement $\theta$. So that the T-Map may be used for metric computations, the units along all axes should be the same, i.e., a length [L]. For that reason, the scale assigned to the axis for angle $\theta$ is made $\theta^{\prime}=\theta d / 2$ where, for the profile in Fig. $2, d=\overline{R S}$.

For any given size for the profile, such as for the MSP, the T-Map is a hypersection of the full 4D T-Map, i.e., a 3D volume.

\section{Profile decomposition and the 3D primitive T-Map for one generic arc-segment}

When a complete line-profile comprising only circular arc-segments is decomposed, the resulting separate arc-segments are joined at points having either $\mathrm{C}^{0}$-continuity (a vertex) or $\mathrm{C}^{1}$-continuity (a common tangent). For each segment, a solid-model T-Map primitive is created in a local coordinate 
frame. Then, the primitive solid models are transformed (displaced and deformed) to be consistent with a common global coordinate frame, after which they are combined by Boolean intersection to identify the region of common points that form the T-Map for the complete line profile.

Although each T-Map primitive is unbounded in one direction, the final T-Map for the entire profile is a bounded space containing all possible mapped points that correspond to the acceptable profile deviations.

Boundaries to the tolerance-zone and to the T-Map have the same function, but they exist in different spaces. The boundaries of a tolerance-zone constrain the displacements of the profile (feature), e.g., the two profiles drawn with dotted lines in Fig. 2, to only those manufacturing deviations that are permitted by a designer's choice for the one or more tolerances that are specified for the profile. In the T-Map space, the boundaries separate points, each corresponding to a profile displacement and size, into acceptable and unacceptable regions. Therefore, there is a one-to-one geometric transformation that relates the boundaries of the tolerance-zone, and every manufacturing variation of the feature within them, to points on the bounding surfaces of the T-Map, and to every point within them.

Although the full T-Map for a line-profile is a 4D solid, the morphology of the 3D hypersections is discontinuous with change in size for generally shaped line-profiles. Therefore, the list of steps below is for the purpose of generating a single 3D hypersection of the 4D T-Map. In the subsections that follow, the method is applied to the MSP. Its application to other allowable sizes of the profile is reserved for section 5.3.

There are six steps to building the solid model for a 3D hypersection of the T-Map for a given-sized line-profile:

1. Decompose the entire line-profile into its $n$ segments,

2. Create the T-Map primitive (3D solid) relative to a local reference system $O x_{i} y_{i}(i=1,2, \ldots, n)$ for each of the segments,

3. Identify a global reference $x^{\prime} y^{\prime}$-frame for the entire MSP and transform each 3D T-Map primitive to this frame,
4. Intersect these transformed T-Map primitives in this global reference frame to get a tentative 3solid T-Map (hypersection) for the entire profile,

5. (Optional) Identify the invariant point (pole) (Hain, 1967; Davidson and Shah, 2012) corresponding to the greatest allowable rotation of the MSP, and

6. (Optional) Transform the tentative T-Map to its representation in a canonical global $x y$-frame with its origin at the pole.

In the next three subsections we apply the first two of these steps to the MSP of a generic arc-segment, and, in sections 5.1 and 5.2, we apply all six to the arc-slot in Fig. 1a.

\subsection{T-Map primitive for the middle-sized profile (MSP) of a generic arc-segment}

A generic arc-segment $\overline{A B}$ is shown in Fig. 3a with its MSP (arc with a dashed line) and the boundaries to its tolerance-zone (arcs with solid lines), all represented in a coordinate frame having its origin at the common arc-center. The orientation of this coordinate frame is unimportant, but its center must be at the common center. The MSP may be approximated by a continuous set of tangent line-segments (five are shown) that range over the angle of the arc. Manufacturing variations from part to part permit each such line-segment to be located radially from $-t / 2$ to $+t / 2$, but tolerance $t$ offers no limit to its tangential location.

Small translated locations are represented in the T-Map space with coordinates $e_{x}$ and $e_{y}$ that correspond to the $x$ - and $y$-directions in Fig. 3a. Therefore, in the 2D T-Map space, all allowable translated locations for any one of the five line-segments in Fig. 3a lie in the interior region between its corresponding parallel boundaries that are separated by tolerance $t$. This region is unbounded in the direction of the line-segment. The Boolean intersection of two of these is the rhombus shown with the speckled shading in Fig. 3b. The intersection of five of these, one for each of the short line-segments in Fig. 3a tangent to the MSP, is the smaller hatched region in Fig. $3 b$. Then, when the arc-segment $\overline{A B}$ is represented by a continuous array of tangents, i.e., as an envelope, the boundary of the area also becomes continuous (Fig. 3c). Since the body on which the manufactured arc-profile is produced is rigid and not displacing or deforming, each point in this hatched area of the T-Map space represents one allowable perfect-form 
manufacturing outcome (displaced location) for the middle-sized arc-profile in Fig. 3a relative to its theoretical location on any one part. The entire hatched area in Fig. 3c corresponds to all of its allowable translated locations. For example, the two translated locations $C$ and $D$ of the MSP in Fig. 3a (dotted lines) are represented with corresponding points $C$ and $D$ at the T-Map boundary in Figs. 3b and 3c.


(d)

Fig. 3 (a) The middle-sized profile for a generic arc-segment (dashed-lined arc) in the (exaggerated) tolerance-zone that is specified with the profile tolerance $t$; two perfect-form variations $C$ and $D$ (dotted lines); (b) The approximated 2D T-Map; (c) The 2D T-Map with a continuous boundary; (d) The 3D T-Map primitive

Although the boundaries to the tolerance-zone in Fig. 2 limit the rotation of the MSP of the entire profile to $t / \overline{A B}$ (see section 3), the boundaries for the generic arc-segment in Fig. 3a provide no limit whatever to its rotated location about local origin $O$, a property that corresponds to the collective unlimited tangential translated locations of the approximating line-segments in Fig. 3a. Therefore, the 3D T-Map primitive for the arc-segment is formed by extruding the hatched area in Fig. $3 \mathrm{c}$ in the orthogonal direction to the $e_{x} e_{y}$-plane, i.e., in the Cartesian $\theta^{\prime}$-direction (Fig. 3d). The result is an open right rhombic prism with one pair of opposite cylindrically rounded edges that possess $\mathrm{C}^{1}$-continuity with the adjoining planar surfaces. Since the prism and cylindrical shells have a common axis, we call this combined T-Map shape a 'cylism'.

\subsection{T-Map primitive for other sizes of the generic arc-segment}

Certainly there are manufacturing variations of the arc-slot in Figs. 1 and 2 for which all arc-segments would be further inward or outward from the MSP. To determine the impact of this fourth variable (section 3) for size on the T-Map primitive, the generic arc and tolerance-zone boundaries in Fig. 3a are redrawn in Fig. 4a to include such an arc $\overline{A^{\prime} B^{\prime}}$ (long-and-short dashed line); it is larger than its MSP by the amount $\Delta F$.
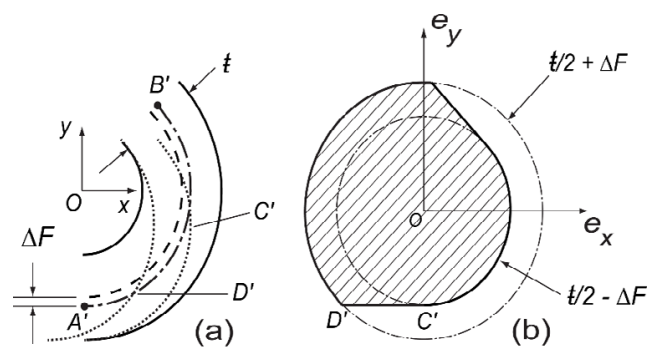

Fig. 4 (a) A profile (long-and-short dashed arc) for the generic arc-segment which is larger than the MSP by $\Delta F$; two perfect-form variations $C^{\prime}$ and $D^{\prime}$ (dotted ares); (b) Its 2D T-Map primitive

Tangent line-segments to arc $\overline{A^{\prime} B^{\prime}}$ may only translate radially outward by the amount $t / 2-\Delta F$, but they can translate inward by $t / 2+\Delta F,-t / 2 \leq \Delta F \leq t / 2$, so generating several sets of parallel lines separated by $t$, in the same manner used to construct Fig. 3b. The Boolean intersection of a continuous array of limiting parallel tangents around arc $\overline{A^{\prime} B^{\prime}}$ generates the $2 \mathrm{D}$ T-Map primitive (hatched region) in Fig. 4b. Just as in Fig. 3c, each point in this hatched area of the T-Map space represents one allowable perfect-form translational displacement in Fig. 4a of the arc of increased size, and the entire area corresponds to all of its allowable translated locations. Two examples are the translated locations $C^{\prime}$ and $D^{\prime}$ (dotted arcs in Fig. 4a) which are represented with corresponding points $C^{\prime}$ and $D^{\prime}$ in Fig. $4 \mathrm{~b}$.

Once again, there is no limit to rotation of the larger-sized arc $\overline{A^{\prime} B^{\prime}}$ about $O$, so its 3D T-Map primitive is also formed by extruding the hatched area in Fig. $4 \mathrm{~b}$ in the Cartesian $\theta^{\prime}$-direction orthogonal to the $e_{x} e_{y}$-plane. The result is an asymmetrical open right 'cylism' with one pair of opposite cylindrically 
rounded edges. One rounded edge possesses $\mathrm{C}^{1}$-continuity with the adjoining planar surfaces, but the other may have $\mathrm{C}^{0}$-continuity (e.g., the 'cylism' formed from the shape in Fig. 4b) or $\mathrm{C}^{1}$-continuity, depending on the value for $\Delta F$ and the included angle of the arc.

\subsection{T-Map primitive referred to a different origin: the shear matrix}

When comparing with Fig. 3, it is clear that the local coordinate frames in Fig. 2 for the four arc-segments of the arc-slot have origins at distinct points $O, R$, and $S$. Yet, to obtain the Boolean intersection of the four T-Map primitives, all arcs must be represented in the same coordinate frame. The need, then, is to transform any arbitrary allowable displacement of a profile arc-segment $i(i=1,2,3,4)$, represented in its local reference frame $O x_{i} y_{i}$ (Figs. 3a and $4 \mathrm{a}$ ), to the equivalent representation in a global reference frame $G x^{\prime} y^{\prime}$ (Fig. 5a).

Allowable feature deviations from a theoretical location are two or more orders of magnitude smaller than corresponding dimensions (see (Davidson and Shah, 2012) for a discussion of exceptions). Consequently, they may be treated as differential quantities, and they combine and transform with the same properties as kinematic velocities. This connection to kinematics permits relationships among deviations (small displacements) to be represented with a three-link serial linkage that carries a moveable lamina. There are two identical and juxtaposed laminae in Fig. 5a, and they each contain the following etched features: the $50^{\circ}$ profile arc-segment $\overline{A B}$ (dashed line), local coordinate frame origin $O$, global coordinate frame origin $G$, link $O B$, and pole $P$.

The moveable lamina is initially coincident with the fixed one, i.e., with the dashed-lined representation for arc $\overline{A B}$ and length $\overline{O B}$. It is then displaced by a small amount that is represented in the $O x_{i} y_{i}$-frame both with translation vector $\boldsymbol{e}_{O}$ and (small) rotation $\theta$. At this displaced location of the moveable lamina, length $\overline{O B}$ is shown with a solid line and arc $\overline{A B}$ with a dotted line. Points $P$ on both laminae are coincident, so $P$ is the pole, or invariant point, for displacement $\left(\boldsymbol{e}_{O}, \theta\right)$.

Note that, to provide a compact layout to Fig. 5, angle $\theta$ (direction $\mathrm{CW}$ ) and values for components $e_{x i}^{\prime}$ and $e_{y i}^{\prime}$ of displacement are all negative.



Fig. 5 (a) A middle-sized arc-segment at its theoretical location (dashed-lined arc) and at an (exaggerated) allowable small displacement (dotted arc) that is represented both in its local coordinate frame $O x_{1} y_{1}$ and global frame $G x^{\prime} y^{\prime}$ located at general point $G$; (b) The extracted vector triangle for small displacements

From kinematics, small displacements at two points $O$ and $G$ on a moving lamina are related by

$$
\boldsymbol{e}_{G}=\boldsymbol{e}_{O}+\boldsymbol{e}_{O G}
$$

in which $\boldsymbol{e}_{O G}=\boldsymbol{\theta} \times \boldsymbol{c}$ (Fig. 5) is the small displacement of $G$ relative to $O$, i.e., as perceived by an observer at $O$ and in fixed frame $O x_{i} y_{i}$. Relative displacement $\boldsymbol{e}_{O G}$ is directed at right angles to $\overline{O G}$ in a sense consistent with $\theta$, and with the sense and direction for the corresponding angular displacement of the moving lamina (Fig. 5b). Of course, to effect the addition in Eq. (1), all three vectors must be represented in the same frame of reference. Typically, Eq. (1) is applied (Uicker et al., 2010) with all vectors represented in fixed frames of reference that have their coordinate axes parallel to those in $O x_{i} y_{i}$, and the origin of each such frame is instantaneously coincident with one labeled point of the linkage. Instead, however, we choose to represent the vectors in frames that are parallel to the global $G x^{\prime} y^{\prime}$-frame (Fig. 5a), but usually have distinct origins. This requires first representing the vectors $\boldsymbol{e}_{O i}$ in rotated local $x_{i}^{\prime} y_{i}^{\prime}$-frames $(i=1,2,3,4)$ that have been rotated to be parallel to the global $G x^{\prime} y^{\prime}$-frame (Fig. 5a); the resulting vectors $\boldsymbol{e}_{O i}^{\prime}$ may then be used in $\boldsymbol{e}_{G}{ }_{G}=\boldsymbol{e}^{\prime}{ }_{O}+\boldsymbol{e}^{\prime}{ }_{O G}$ to relate 
displacements. The first two scalar equations in the transformation

$$
\begin{aligned}
& \left.\left[\begin{array}{c}
e_{x i}^{\prime} \\
e_{y i}^{\prime} \\
\theta
\end{array}\right]=\left[\begin{array}{ccc}
1 & 0 & -c_{y} \\
0 & 1 & c_{x} \\
0 & 0 & 1
\end{array}\right]\left[\begin{array}{ccc}
\mathrm{C} \phi_{i} & -\mathrm{S} \phi_{i} & 0 \\
\mathrm{~S} \phi_{i} & \mathrm{C} \phi_{i} & 0 \\
0 & 0 & 1
\end{array}\right]\left[\begin{array}{c}
e_{x i} \\
e_{y i} \\
\theta
\end{array}\right]\right\} \\
& =\boldsymbol{S R}\left[\begin{array}{lll}
e_{x i} & e_{y i} & \theta
\end{array}\right]^{\mathrm{T}}
\end{aligned}
$$

represent both operations, and the third equation acknowledges that small rotation $\theta$ is the same everywhere on the moving lamina (Note that $\mathrm{C} \phi_{i}=\cos \phi_{i}$, etc.).

By not combining the matrices in Eq. (2), we preserve the uncoupled operations of rotation of each vector $\boldsymbol{e}_{O i}$ to its equivalent $\boldsymbol{e}_{O i}^{\prime}$, represented by the rigid-body rotation $\boldsymbol{R}$, from the small-displacement relation in Eq. (1), which is represented exclusively by the matrix $S$ and its corresponding vector triangle (e.g., Fig. 5b). An important consequence of this equivalence between Eq. (1) and matrix $S$ is that $S$ may be used only to transform information in Eq. (1) between frames that have distinct origins but all corresponding axes parallel, such as between the $O x_{i}^{\prime} y_{i^{-}}$and $G x^{\prime} y^{\prime}$-frames in Fig. 5.

The coupling of a translation $\boldsymbol{e}$ and rotation $\theta$ in matrix $\boldsymbol{S}$ causes shear of the right 'cylismic' T-Map shapes, which are described in sections 4.1 and 4.2 (e.g., Fig. 3d), to oblique 'cylisms' in which the central axis has the direction-ratios $e_{x}: e_{y}: \theta^{\prime}::-c_{y}: c_{x}: d / 2$ and in which all cross-sections parallel to the $e_{x} e_{y}$-plane in a T-Map are preserved. Typical crosssections are the hatched regions in Figs. $3 \mathrm{c}$ and $4 \mathrm{~b}$.

Since the shear matrix $S$ in Eq. (2) is a kinematic transformation, angle $\theta$ has no units. Therefore, when $\boldsymbol{S}$ is used in Eq. (2) to transform coordinates of points $\left(\boldsymbol{e}_{O}, \theta^{\prime}\right)$ of a T-Map from a rotated local frame (e.g., the $O x_{i}^{\prime} y_{i}^{\prime}$-frame in Fig. 5a) to a global frame $G x^{\prime} y^{\prime}$, angle $\theta$ must first be extracted from $\theta^{\prime}=\theta d / 2$ (the end of section 3). As a result of this relation, $\boldsymbol{S}$ may be used to transform both points $\left(\boldsymbol{e}_{O}, \theta^{\prime}\right)$ of the T-Map for a line-profile and small displacements $\left(\boldsymbol{e}_{O}, \theta\right)$ of the arc-segment, between any two coordinate frames having distinct origins but all corresponding axes parallel.

It is helpful to note that shear matrix $\boldsymbol{S}$ may also be used to transform points of an entire T-Map from one global frame (e.g., the $G x^{\prime} y^{\prime}$-frame in Fig. 5a) to another, say the $H x^{\prime} y^{\prime}$-frame (not shown), that has a distinct origin but axes parallel to the first. See the end of section 5.2.

\section{Line-profile example: the arc-slot in Fig. 1a}

The MSP of the arc-slot in Figs. 1a and 2 is redrawn in Fig. 6 showing its decomposition into the four arc-segments 1, 2, 3, and 4. The local coordinate frames $O x_{i} y_{i}(i=1,3), R x_{2} y_{2}$, and $S x_{4} y_{4}$, are also shown at the respective local arc-centers for these segments and, in each case, with the $y$-axis lying in the plane of bilateral symmetry for the arc-segment.

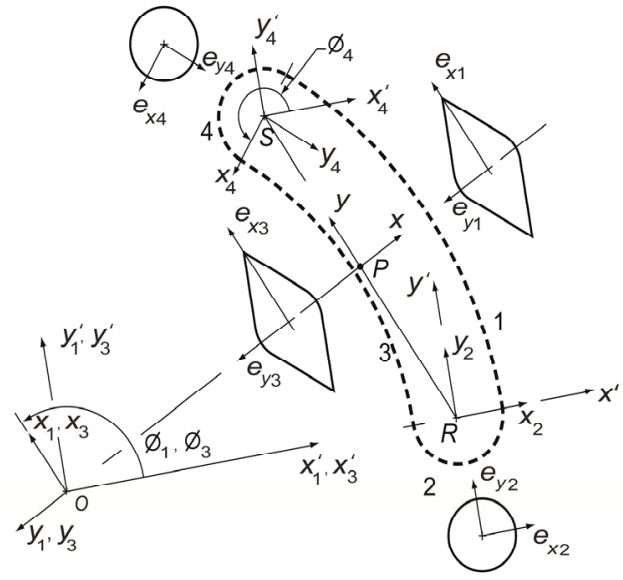

Fig. 6 Global $R x^{\prime} y^{\prime}$ - and $P x y$-frames for the arc-slot in Fig. 1a which has been decomposed into four arcsegments, each with its local reference frames $x_{i} y_{i}$ and $x_{i}^{\prime} y_{i}^{\prime}$ and its 2D T-Map primitive. The angles $\phi_{i}$ shown are for global frame $R x^{\prime} y^{\prime}$

When the two parts in Fig. 1 are assembled, one important design requirement is that the pin $\overline{R S}$ of the adjusting-link not bind over the entire arc $\overline{R S}$ of the casting. Consequently, we have chosen the $R x^{\prime} y^{\prime}$-frame as an important global frame of reference in which to describe the T-Map of the entire line-profile; its $x^{\prime}$-axis is chosen to align with the center distance between pins of the adjusting link when it is at the lower end $(R)$ of the slot. This line-profile T-Map would be used in a tolerance loop that also would include (a) the size, position, and orientation tolerances for the hole at $O$ in the casting, and (b) the size, relative position, and orientation tolerances for the pins on the adjusting-link. The same 
tolerance-loop would occur with a radial alignment of a global $x^{\prime} y^{\prime}$-frame having its origin at point $S$.

Of course, the local $x_{i}^{\prime} y_{i}^{\prime}$-frames $(i=1,2,3,4)$ in Fig. 6 are obtained from the local $x_{i} y_{i}$-frames with the rotation matrix $\boldsymbol{R}$ in Eq. (2). Note that, to be consistent with Eq. (2), the values for $\phi_{i}, c_{x i}$, and $c_{y i}$ in $\boldsymbol{R}$ and $\boldsymbol{S}$ must be chosen in the following manner: angle $\phi_{i}$ is measured from the global frame of interest (either $R x^{\prime} y^{\prime}$ or Pxy in Fig. 6) to the local $x_{i} y_{i}$-frame, vector $c$ is directed from the local to the global origin, and $c_{x i}$ and $c_{y i}$ are projections of this vector $c$ onto the global axes. The 2D T-Map primitives are shown in Fig. 7a for the four arc-segments.

\subsection{D T-Map for the MSP in global frame $R x^{\prime} y^{\prime}$}

When a line-profile is part of a tolerance loop for which a worst-case tolerance analysis is desired, it is the 3D T-Map for the MSP that is required for the analysis because the positional and orientation deviations are greatest for this size (section 5.1 in (Davidson and Shah, 2012)). Further, the functional requirement for a profile is often constructed from position and orientation requirements, rather than from requirements for size deviations. Therefore, we first confine attention to the 3D T-Map for the MSP for the arc-slot in Fig. 1a. Further, from the functional requirements for an assembly of the two parts in Fig. 1 (section 5), we choose, as a practical example, to formulate this T-Map in global frame $R x^{\prime} y^{\prime}$. T-Maps for larger and smaller sizes of the line-profile will be addressed in section 5.3.

The 2D primitives for all four arc-segments are shown in Fig. 7a arranged relative to an $x y$-frame that is aligned with the axis of symmetry for each. Then, in Fig. 6, they are separated, and each one is shown deployed around the profile with an orientation and alignment that is consistent both with its own local $x_{i} y_{i}$-frame and the single $x y$-frame in Fig. $7 \mathrm{a}$.

To construct the T-Map in $R x^{\prime} y^{\prime}$ for the arc-slot in Figs. $1 \mathrm{a}$ and 2 , values for $\phi, c^{\prime}{ }_{x}$, and $c^{\prime}{ }_{y}$ are identified from the five coordinate frames $\left(R x^{\prime} y^{\prime}\right.$ and $x_{i} y_{i}$-frames, $\left.i=1,2,3,4\right)$ and the geometry shown in Fig. 6. The values are listed in Table 1 by using the rules established at the end of section 5 . These values, when used in matrices $\boldsymbol{R}$ and $\boldsymbol{S}$ in Eq. (2), constitute Step 3 in the procedure described at the end of section 4. The geometric functions required in the procedure are accomplished on the T-Map primitives with the
ACIS software (Spatial Co., 2012) and can be keyed to the steps listed in section 4: extrusion of the 2D primitives in Fig. 7a to form right cylinders and cylisms; rotation $\boldsymbol{R}$ and shear $\boldsymbol{S}$ of these to form the operand T-Map primitives (oblique cylinders or 'cylisms'), all in one global reference frame $R x^{\prime} y^{\prime}$; and finally the Boolean intersection of the operands to form the T-Map in $R x^{\prime} y^{\prime}$ for the entire arc-slot. The result is the $3 \mathrm{D}$ hypersection shown in Fig. 8 comprising segments of just two circular cylindrical shells: a right-circular one formed from the T-Map primitive for arc-segment 2 of the arc-slot, and an oblique one formed from arc-segment 4.

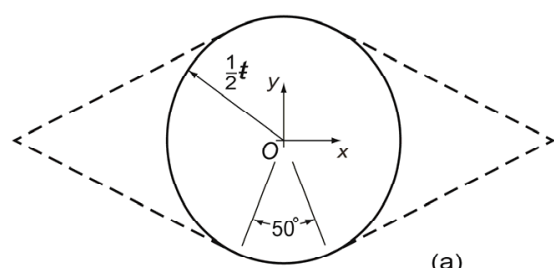

(a)

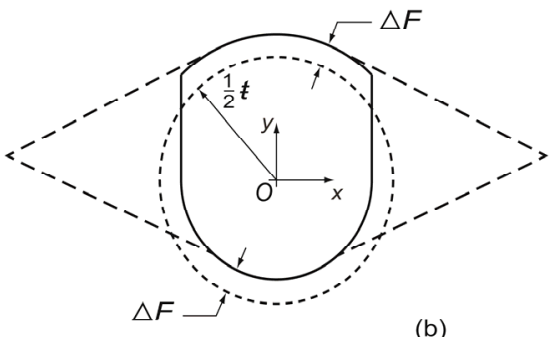

(b)

Fig. 7 2D T-Map primitives for the arc-segments of the curved slot in Figs. 1a and 2, the dashed lines (rounded rhombus) for both arcs centered at $O$ and the solid lines for the two arcs at $R$ and $S$; (a) For the MSP; (b) For arcs 1,2 , and 4 of an arc-slot larger than the MSP by $\Delta F$

Table 1 Values to formulate $R$ and $S$ for the T-Map represented in global frame $R x^{\prime} y^{\prime}$

\begin{tabular}{cccc}
\hline Arc-segment & $\phi\left(^{\circ}\right)$ & $c^{\prime}{ }_{x}(\mathrm{~mm})$ & $c_{y}^{\prime}(\mathrm{mm})$ \\
\hline 1 & 115 & 68.00 & 0 \\
2 & 0 & 0 & 0 \\
3 & 115 & 68.00 & 0 \\
4 & 230 & 24.29 & -52.09 \\
\hline
\end{tabular}

Since $c_{x 2}^{\prime}=c^{\prime}{ }_{y 2}=0$ (Table 1), the 2D T-Map primitive for arc-segment 2 is not sheared, and so, remains a right circular cylinder with axis $O \theta^{\prime}$ in the T-Map space. The other cylinder is oblique and derives from arc-segment 4 . The direction ratios for 
its axis may also easily be obtained from the values in Table 1, i.e., $-c_{y}: c_{x}: d / 2:: 52.09: 24.29: 28.74$. Since the values for $c^{\prime}{ }_{x}$ and $c^{\prime}{ }_{y}$ are the same for arc-segments 1 and 3, their T-Map primitive is a single oblique cylinder in $R x^{\prime} y^{\prime}$ that is doubly traced. Recalling that $d / 2=68 \sin 25^{\circ}=28.74 \mathrm{~mm}$ (Fig. 1 a and the penultimate paragraph of section 3 ), the direction ratios for its common axis are $e_{x}^{\prime}: e_{y}^{\prime}: \theta^{\prime}::-c_{y}: c_{x}: d / 2:: 0: 68.00: 28.74$, indicating that the axis lies in the $e_{y}^{\prime} \theta^{\prime}$-plane of the T-Map. After Boolean intersection, the doubly traced cylinder from arc-segments 1 and 3 is trimmed away, its only remnants being two portions of the circular edge of diameter $\boldsymbol{t}$ in the $e_{x} e_{y}$-plane and the two surface-lines shown in Fig. 8. The two lines, one in front and the other hidden, represent the tangency of planes from the 'cylisms' for segments 1 and 3, after their extrusion and shear from the dashed lines in Fig. 7a.

The dominance of arc-segments 2 and 4 in controlling displacements of the MSP in Fig. 2 may also be verified intuitively. Suppose that arc-segments 1 and 3 were removed from the profile, so that the profile were defined only by the bar $\overline{R S}$ and two rigidly attached semicircular cams, one at each end. These cams would provide the entire array of constraints experienced by the complete line-profile in Fig. 2 because the arcs 2 and 4 are semicircles. True, for radial translated locations within the $50 \mathrm{deg}$ arc for segments 1 and 3, a profile variation (MSP or one of different size) would also touch a boundary-arc of the tolerance zone for segment 1 or 3 , but it would add no additional constraint. This touching corresponds to the remnant circular edge in the T-Map for arcsegments 1 and 3 .

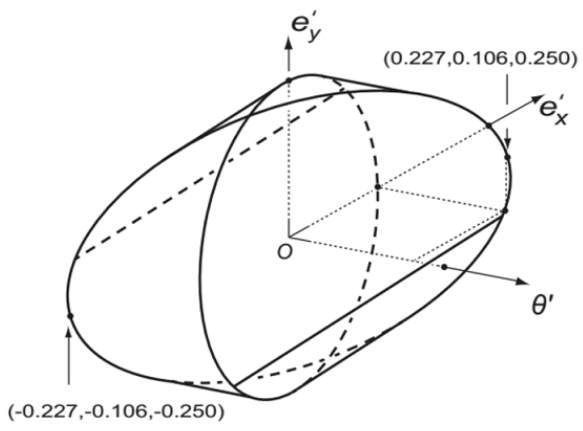

Fig. 8 Boolean intersection of the T-Map primitives which forms the 3D hypersection T-Map in global frame $R x^{\prime} y^{\prime}$ for the MSP of the arc-slot in Fig. 1a. The coordinate values correspond to the MSP rotated to its two limits in the tolerance-zone, e.g., the CCW limit shown in Fig. 2. The diameter of the circular edge is $t$
When the 3D hypersection T-Map is created for a reference frame having its origin at a general location, the shear matrix $\boldsymbol{S}$ induces coupling between $\theta^{\prime}$ and coordinates $e_{x}$ and $e_{y}$ of the T-Map, so amplifying the values of $e_{x}$ and $e_{y}$ that would be used in a tolerance accumulation (stackup) for an assembly. This amplification is important in practical applications, such as the radial variation $e_{x}^{\prime}$ through point $R$ (Fig. 3) in the assembly of the two parts in Fig. 1. It is similarly important when applying T-Maps to tolerance accumulation with features other than profiles. For instance, for planar features, the same shearing of a canonical T-Map is required when a stackup includes a part having an offset between two planar faces that are included in the tolerance loop (e.g., (Davidson et al., 2002)).

\subsection{D T-Map for the MSP in the canonical global frame $P_{x y}$}

When the T-Map for a line-profile is constructed with a reference frame having its origin at pole $P$, the displacements $e_{x}, e_{y}$, and $\theta$ are uncoupled because, for the profile rotated to its limit, $\theta_{\max }$, no translation at $P$ occurs. The pole $P$ as origin is sufficient to identify the canonical frame of reference for the T-Map when the line-profile does not exhibit a bilateral line of symmetry. However, when there is such a line, one of its coordinate axes should be aligned with the axis of symmetry. Consequently, we have chosen the canonical Pxy-frame in Fig. 6 with its origin at the pole (see section 4.3 and step 5 in section 4 ) for the arc-slot and axis $P x$ aligned with the axis of bilateral symmetry.

Construction of the 3D hypersection for the MSP in frame Pxy follows the same steps (section 4) as those used in constructing the T-Map in the $R x^{\prime} y^{\prime}$-frame (section 5.2). The revised values for $\phi, c_{x}$, and $c_{y}$ are listed in Table 2 for the four arc-segments comprising the line-profile. Note that all $\phi_{i}$-values are $25^{\circ}$ less than those in Table 1 for all arc-segments, so indicating that the matrix $\boldsymbol{R}$ in Eq. (2) causes the rotated local frames of reference (not shown in Fig. 6) to be aligned properly with the Pxy-frame.

Table 2 Values to formulate $R$ and $S$ for the T-Map represented in global frame $P x y$

\begin{tabular}{cccc}
\hline Arc-segment & $\phi\left(^{\circ}\right)$ & $c_{x}(\mathrm{~mm})$ & $c_{y}(\mathrm{~mm})$ \\
\hline 1 & 90 & 61.63 & 0 \\
2 & -25 & 0 & 28.74 \\
3 & 90 & 61.63 & 0 \\
4 & 205 & 0 & -28.74 \\
\hline
\end{tabular}


The 3D hypersection T-Map, created for the MSP in the $P x y$-frame (Fig. 9), is once again formed from the intersection of two oblique circular cylinders, now with both axes in the $e_{x} \theta^{\prime}$-plane. These correspond to the T-Map primitives from arc-segments 2 and 4, and, since the direction ratios (Table 2) are $e_{x}: e_{y}: \theta^{\prime}::-c_{y}: c_{x}: d / 2:: \pm 28.74: 0: 28.74$, the axes are at right angles.

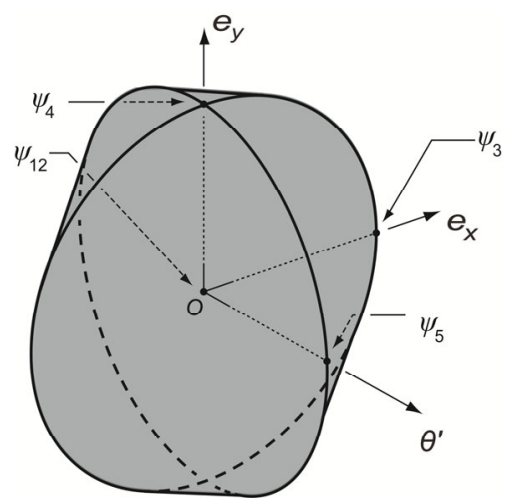

Fig. 9 T-Map of Fig. 8 for the MSP of the arc-slot in Fig. 1a, now transformed to the canonical global $P x y$-frame. Both circular edges have diameter $t$

After intersection, these T-Map primitives form the 3D hypersection shown in Fig. 9. It is a square elliptic globe that is inscribed in a sphere of diameter t; it is one form of generalized Archimedean globe (Appendix A and Apostol and Mnatsakanian, 2012). The doubly traced cylinder from arc-segments 1 and 3 is only present as two portions of the circular edge in the $e_{x} e_{y}$-plane and the two parallel surface-lines that lie in the $e_{x} \theta^{\prime}$-plane and at $45^{\circ}$ to the $e_{x}$ - and $\theta^{\prime}$-axes.

Often the location of pole $P$ is not known $a$ priori, as it is for the symmetrical arc-slot in Figs. 1a, 2 , and 6. For such line-profiles, transformation $\boldsymbol{S}$ may be applied to that point $\left(e_{x}^{\prime}, e_{y}^{\prime}, \theta_{\text {max }}^{\prime}\right)$ of the complete T-Map for which coordinate $\theta^{\prime}$ is greatest. Then, since $P$ is stationary, the outcome coordinates in Eq. (2), $\left(e_{x}, e_{y}, \theta\right)$, can be set to $(0,0, \theta)$. As one example, when the coordinates at a $\theta^{\prime}$-limit in Fig. 8 are substituted in Eq. (2), the relation

$$
\left[\begin{array}{l}
0 \\
0 \\
\theta
\end{array}\right]=\left[\begin{array}{ccc}
1 & 0 & -c_{y} \\
0 & 1 & c_{x} \\
0 & 0 & 1
\end{array}\right]\left[\begin{array}{c}
0.227 \\
0.106 \\
0.0087
\end{array}\right]
$$

results. Of course, to use matrix $\boldsymbol{S}$ in Eq. (2) properly, angle $\theta_{\max }$ must be obtained from $\theta^{\prime}=\theta d / 2$, i.e., 0.250 $=28.74 \theta$, where $d / 2=28.74 \mathrm{~mm}$ is the characteristic length for the line-profile. The results are $\left(c_{x}, c_{y}\right)=$ $(-12.15,26.05) \mathrm{mm}$. When locating the pole $P$ with this procedure, it is important to note that the T-Map will then be represented in a global $P x^{\prime} y^{\prime}$-frame (not shown in Figs. 2 and 6) with its axes parallel to those in frame $R x^{\prime} y^{\prime}$ because matrix $\boldsymbol{S}$ is confined solely to translational changes in location of origin. An additional rotation about the $\theta^{\prime}$-axis is required for its representation in the canonical frame $P x y$.

\subsection{D T-Map for the line-profile in the canonical global frame Pxy}

The six steps listed in section 4 may also be modified appropriately for construction of 3D hypersections of the 4D T-Map of a line-profile for increments in size larger or smaller than the MSP by any amount $\Delta F \leq t / 2$. The first modification to the steps is to use the 2D T-Map primitives from Fig. 7b that exhibit less symmetry than those in Fig. 7a. When these are deployed at the individual arc-segments around the profile (Fig. 10), it is necessary to recognize that larger size of the entire profile corresponds to a decrease in radius for the concave arc-segment 3.



Fig. 10 The same geometry as in Fig. 6, but now the deployed 2D T-Map primitives are for a profile size $\Delta F$ larger (long-and-short dashed curve) than the MSP (Fig. 7b)

The complete $4 \mathrm{D}$ construction is undertaken by using sufficient values of allowable increments $\Delta F$ in size to identify all the changes in morphology (number and orientation of both faces and cylindrical shells) that occur over the allowable size range $-t / 2 \leq$ 
$\Delta F \leq t / 2$. Of course, all such 3D T-Maps must be formed in steps 3 and 4 with the same global frame that is used for the MSP T-Map, e.g., global frame $R x^{\prime} y^{\prime}$ or $P x y$. An example of a 4D T-Map is shown in Fig. 11; it is represented in frame Pxy. Careful sketching of a larger sized profile within the boundaries reveals that arc-segments 1 and 3 now provide constraint because contact of the larger profile at the inner boundary at segments 2 or 4 does not occur. Therefore, as size increases from the middle size, planar faces evolve from the tangent lines to cylinders in the T-Map for the MSP (e.g., in Fig. 8).

The practical use for any 4D T-Map for a line-profile is in conducting a statistical tolerance analysis, because each point in the 4D T-Map contributes to the sample-space of possible deviations for the profile.

\section{Conclusions}

This paper introduces a new method for constructing T-Maps for line-profiles that are to be manufactured on rigid parts. A T-Map represents a designer's specified limits to the allowable geometric variations to the profile manufacturing. The new method constitutes a decomposition of the profile into joined segments, the formation of a T-Map primitive (a 3D solid) for each one in a local coordinate system, the transformation of all of these to different orientations and shapes in a common reference frame, and the Boolean intersection of these transformed T-Map primitives to obtain the T-Map for the entire profile. All of these geometric operations are normally found in computer-implementations of computer-aided geometric design (CAGD).

Although there are other ways (He et al., 2013) to arrive at Eqs. (1) and (2), in this paper we have used a kinematic equivalent to represent the allowable displacements of the perfect-form profile within its tolerance-zone.

The authors use an arc-slot profile (for adjustment) as an example to explain the procedures of construction. Certainly, this is a special case, but the procedures in the body of the paper are general and apply to any shape of profile that is formed from circular arc-segments: closed or open, and concave or convex. Further, current work will expand this method to line-profiles containing free-form segments, thereby providing potential for its use in generating T-Maps for line-profiles of any shape.

\section{Acknowledgements}

The authors acknowledge the efforts of Mr. Shyam RAO and Mr. Nathan KALISH for preparing the figures at the Design Automation Laboratory at Arizona State University, USA.

\section{References}

ASME (American Society of Mechanical Engineers), 2009. Dimensioning and Tolerancing, ASME Y14.5. ASME, New York.

Ameta, G., Samper, S., Giordano, M., 2011. Comparison of spatial math models for tolerance analysis: ToleranceMaps, deviation domain, and TTRS. ASME Journal of Computing and Information Science in Engineering,



Fig. 11 4D T-Map in global frame $P x y$, now including the manufacturing variation of size. No additional changes in morphology occur toward $\psi_{1}$ and $\psi_{2}$, i.e., for both $-t / 2 \leq \Delta F \leq-t / 4$ and $t / 4 \leq \Delta F \leq t / 2$ 
11(2):021004. [doi:10.1115/1.3593413]

Apostol, T.M., Mnatsakanian, M.A., 2012. New Horizons in Geometry. Mathematical Association of America, Washington, DC.

Davidson, J.K., Shah, J.J., 2012. Modeling of geometric variations for line-profiles. ASME Journal of Computing and Information Science in Engineering, 12(4):041004. [doi:10.1115/1.4007404]

Davidson, J.K., Mujezinović, A., Shah, J.J., 2002. A new mathematical model for geometric tolerances as applied to round faces. ASME Journal of Mechanical Design, 124(4): 609-622. [doi:10.1115/1.1497362]

Davidson, J.K., Shah, J.J., Mujezinović, A., 2005. Method and Apparatus for Geometric Variations to Integrate Parametric Computer-aided Design with Tolerance Analysis and Optimization. US Patent No. 6963824, Washington, DC.

Giordano, M., Duret, D., 1993. Clearance space and deviation space: application to three-dimensional chains of dimensions and positions. In: Bourdet, P., Mathieu, L. (Eds.), Proc. 3rd CIRP Seminar on Computer-aided Tolerancing, Eyrolles, Paris, p.179-196.

Giordano, M., Pairel, E., Samper, S., 1999. Mathematical representation of tolerance zones. In: van Houten, F., Kals, H. (Eds.), Global Consistency of Tolerances. Springer, Amsterdam, p.177-186. [doi:10.1007/978-94-017-1705-2 -18]

Hain, K., 1967. Applied Kinematics, 2nd Edition. McGraw-Hill, New York.

He, Y., Davidson, J.K., Shah, J.J., 2013. Tolerance-Maps for line-profiles constructed from Boolean operations on primitive T-Map elements. 33rd ASME Computers and Information in Engineering Conference, Portland, OR. ASME, New York, USA, p.V02AT02A003. [doi:10. 1115/DETC2013-12393]

ISO (International Organization for Standardization), 1987. Technical Drawings-Dimensioning and Tolerancing of Profiles, ISO 1660. ISO, Geneva.

Mujezinović, A., Davidson, J.K., Shah, J.J., 2004. A new mathematical model for geometric tolerances as applied to polygonal faces. ASME Journal of Mechanical Design, 126(3):504-518. [doi:10.1115/1.1701881]

Pasupathy, T.M.K., Morse, E.P., Wilhelm, R.G., 2003. A survey of mathematical methods for the construction of geometric tolerance zones. ASME Journal of Computing and Information Science in Engineering, 3(1):64-75. [doi:10. $1115 / 1.1572519]$

Roy, U., Li, B., 1999. Representation and interpretation of geometric tolerances for polyhedral objects. II: size, orientation and position tolerances. Computer-Aided Design, 31(4):273-285. [doi:10.1016/S0010-4485(99)00028-7]

Spatial Co., 2012. ACIS, Release: R23. Available from http://doc.spatial.com/index.php/Portal:ACIS [Accessed on Nov. 12, 2014]
Uicker, J.J., Pennock, G.R., Shigley, J.E., 2010. Theory of Machines and Mechanisms, 4th Edition. Oxford University Press, Oxford.

\section{Appendix A: an Archimedean globe and dome}

A circumsolid is any geometric shape that circumscribes a sphere. An Archimedean globe is a circumsolid formed as the union of two or more circular cylinders for which the axes are coplanar (the $e_{x} \theta^{\prime}$-plane in Fig. 9) and intersect at one point. Two Archimedean domes are formed when an Archimedean globe is bisected by its central plane that contains the cylinder axes. The square elliptical globe in Fig. 9 is formed by a dilatation in the $e_{y}$-direction of an Archimedean globe, so causing the two circular cylinders to become elliptic in cross-section and the curved edges of the solid to become circles. More properties, figures, and formulae about generalized Archimedean domes and globes may be found in Chapter 5 of Apostol and Mnatsakanian (2012).

\section{中文概要}

\section{题 目: 弧线段 T-Map 布尔交运算获取线轮廓度 T-Map} 的方法研究

目 的: 为使零件在设计阶段实现公差的自动分配, 研究 线轮廓度在计算机中的表达模型。

创新点: 1. 提出一种新的构建线轮廓度公差 T-Map 图的方 法; 2. 用运动学等效的方法表示理想轮廓公差域 的允许偏差。

方 法: 1. 将零件轮廓分解成多段, 然后分别为每段生成 一个实体模型 T-Map（图 6 和 7)；2. 利用布尔 交运算将所有分段 T-Map 合成一个完整线轮廓 度的 T-Map（图 8)；3. 以弧形短槽为例, 演示 创建线轮廓度的方法步骤。

结 论: 将弧形短槽轮廓分成多段, 先实现每一段的 T-Map, 再利用布尔交实现整体线轮廓度公差的 T-Map 图, 证明该方法在构建任意轮廓的线轮廓 度公差上的有效性。

关键词：几何公差; 线轮廓度; 公差模型; 公差带; 布尔 交 